\title{
Analysis of Noise Effects in Long Semiconductor Optical Amplifiers
}

\author{
Y. Said*, H. Rezig and A. Bouallegue
}

SYS'COM Research Laboratory, TIC Department, National Engineering School of Tunis (ENIT), Tunisia

\begin{abstract}
In this letter, we address one of the essential processes to consider in long Semiconductor Optical Amplifiers (SOAs) analysis, which is the noise. Particularly, we investigate the impact of noise effects on the SOA behavior by measuring the gain, the optical signal to noise ratio and the noise figure, referring to numerical simulations. Although its gain dynamics provide very attractive features of high speed optical signal processing, we show that the noise is strong in long SOAs and can limit the performance of the structure. In order to remedy this, we show that using high bias current at moderate input signal power is recommended.
\end{abstract}

\section{INTRODUCTION}

With the tremendous growth of the Internet and the large increase in traffic demands, which have produced a need for new flexible types of services, the necessity of new generation optical networks for managing the high-speed data flows has appeared. One of the most promising technologies for high bit rate optical networks is that related to the exploitation of the huge potential of the SOA amplifier.

By integrating the amplifier functionality into the semiconductor material, the same basic component can perform many different applications. The major advantages of SOAs that lead to great innovation in optical communication technique are their capability to operate as multifunctional devices, their compactness, miniaturization and integration, their high nonlinearity and their potentially low production cost. Moreover, they can provide integrated functionality of internal switching and routing functions that are required for optical networks.

Wavelength converters [1], optical switches [2], signal regenerators [3] as well as optical logic gates [4], all can be made from SOAs, which will lead to large cost reductions and improved performance in future optical-network equipments. Most of these functionalities are based on the nonlinear effects of SOA as the cross-gain modulation (XGM), the cross-phase modulation (XPM), the four wave mixing (FWM) and the cross-polarization modulation (XPolM), etc.

Although lot of remarkable investigations have been performed using short SOAs, the potential of long and ultralong SOAs for high speed all-optical signal processing hasn't been sufficiently studied yet.

Despite long SOAs require special design of the facets in order to prevent unwanted lasing inside the active region, they have proven to be particularly promising devices to perform many key functions, but not in conventional operations. A very attractive feature identified in the work of Slovak et al. [5] is related to acceleration of the inter-band

*Address correspondence to this author at the SYS'COM Laboratory, National Engineering School of Tunis (ENIT) B.P. 37 Le Belvedere 1002 Tunis, Tunisia; Tel: +216 71875 475; Fax: +216 71872 729;

E-mail: y.said@ttnet.tn; youssef_tn@yahoo.com transitions when SOA length is increased. Moreover, the effect of amplified spontaneous emission (ASE) noise, which is known to limit the SOA performance, may become strong in long devices because each part of the structure contributes spontaneous emission as was mentioned by Bramann et al. in [6].

As noise properties of the optical signal have been the subject of extensive studies [7-10] and are still of interest, we will focus, in this letter, on one of the main processes to consider in SOA analysis, which is the noise effect. Especially, we will concentrate on the impact of noise effects on the behavior and the performance of the long devices. This study is based on analyzing, by numerical simulations, the gain, the Optical Signal to Noise Ratio (OSNR) and the noise figure (NF) of the structure.

\section{THEORY}

The wideband model for a bulk SOA proposed by Connelly in $[11,12]$ is of interest to consider in studying SOAs characterization, then it is adopted in this paper. It is based on the numerical solution of the coupled differential equations for carrier-density rate and photon-flux propagation for both the forward and backward signals and the spectral components of ASE. The traveling-wave equations for the signal fields are written as follows:

$$
\left\{\begin{array}{l}
\frac{d E_{s, k}^{+}(z)}{d z}=\left(-j \cdot \beta_{k}+\frac{1}{2}\left(\Gamma \cdot g_{m}\left(v_{k}, n\right)-\alpha(n)\right)\right) \cdot E_{s, k}^{+}(z) \\
\frac{d E_{s, k}^{-}(z)}{d z}=\left(j \cdot \beta_{k}-\frac{1}{2}\left(\Gamma \cdot g_{m}\left(v_{k}, n\right)-\alpha(n)\right)\right) \cdot E_{s, k}^{-}(z)
\end{array}\right.
$$

Here, the propagation of the optical field is assumed on the positive or negative $\mathrm{z}$-direction $\left(\mathrm{E}_{\mathrm{s}, \mathrm{k}}^{+}, \mathrm{E}_{\mathrm{s}, \mathrm{k}}^{-}\right)$of the SOA structure; $\beta_{\mathrm{k}}$ is the propagation coefficient; $\mathrm{g}_{\mathrm{m}}$ denotes the material gain coefficient; $v_{\mathrm{k}}$ symbolizes the optical frequency of the $\mathrm{N}_{\mathrm{s}}$ signals injected with a power $\mathrm{P}_{\mathrm{in}, \mathrm{k}}$ before coupling loss $\left(\mathrm{k}=1\right.$ to $\left.\mathrm{N}_{\mathrm{s}}\right) ; \alpha$ represents the material loss coefficient.

The equation (1) is subject to the boundary conditions, which are given by [11]: 
$\left\{\begin{array}{l}E_{s, k}^{+}(0)=\left(1-\sqrt{R_{1}}\right) \cdot \sqrt{\frac{\eta_{i n} \cdot P_{i n, k}}{h \cdot v_{k}}}+\sqrt{R_{1}} \cdot E_{s, k}^{-}(0) \\ E_{s, k}^{-}(L)=\sqrt{R_{2}} \cdot E_{s, k}^{+}(L)\end{array}\right.$

The signal amplification also depends on the amount of spontaneously emitted noise from the amplifier. The traveling wave equations for the spontaneous emission are given by $[11]$ :

$$
\left\{\begin{array}{l}
\frac{d N_{j}^{+}(z)}{d z}=\left(\Gamma \cdot g_{m}\left(v_{j}, n\right)-\alpha(n)\right) \cdot N_{j}^{+}(z)+R_{s p}\left(v_{j}, n\right) \\
\frac{d N_{j}^{-}(z)}{d z}=-\left(\Gamma \cdot g_{m}\left(v_{j}, n\right)-\alpha(n)\right) \cdot N_{j}^{-}(z)-R_{s p}\left(v_{j}, n\right)
\end{array}\right.
$$

where $\mathrm{N}_{j}^{+}$and $\mathrm{N}_{\mathrm{j}}^{-}$are, respectively, the spontaneous emission photon traveling in the positive and negative $z$-direction; $\mathrm{R}_{\mathrm{sp}}$ denotes the emission noise coupled into $\mathrm{N}_{j}^{+}$and $\mathrm{N}_{j}^{-}$.

The carrier density at position $\mathrm{z}$ in the SOA structure is determined from the steady-state solution to the rate equation [11]:

$$
\begin{aligned}
\frac{d n}{d z}= & \frac{I}{e \cdot d \cdot L \cdot W}-R(n)-\frac{\Gamma}{d \cdot W}\left(\sum_{k=1}^{N_{S}} g_{m}\left(v_{k}, n\right) \cdot N_{s, k}^{+}(z)\right) \\
& -\frac{\Gamma}{d \cdot W}\left(\sum_{k=1}^{N_{s}} g_{m}\left(v_{k}, n\right) \cdot N_{s, k}^{-}(z)\right) \\
& -\left(\sum_{j=1}^{N_{m}-1} g_{m}\left(v_{j}, n\right) \cdot\left(N_{j}^{+}(z)+N_{j}^{-}(z)\right)\right)
\end{aligned}
$$

I denotes the amplifier bias current, which is assumed to have a uniform distribution across the active region width; $\mathrm{R}$ represents the recombination rate term; e is the electronic charge; $\mathrm{N}_{\mathrm{s}, \mathrm{k}}^{+}$and $\mathrm{N}_{\mathrm{s}, \mathrm{k}}^{-}$are, respectively, the photon rate of the wave in the positive and negative $\mathrm{z}$-direction..

\section{NOISE EFFECTS IN A SOA STRUCTURE}

One of major processes to consider in SOA analysis is the amplified spontaneous emission noise, because it strongly affects the structure performance. So, the injected signal and the ASE noise interact nonlinearly as they propagate along the SOA structure. Then, the interaction correlates different spectral components of the noise.

Consequently, we can distinguish three types of noise which are the shot noise, the signal-spontaneous beat noise and the spontaneous-spontaneous beat noise.

The power of the ASE noise generated internally within the SOA is given by:

$$
P_{A S E}=2 \cdot n_{s p} \cdot h \cdot v \cdot(G-1) \cdot B_{0}
$$

where $G$ is the gain at the optical frequency $v, h$ represents the Planck's constant, $B_{0}$ is the optical bandwidth and $n_{s p}$ refers to the population inversion factor. For an ideal amplifier, $\mathrm{n}_{\mathrm{sp}}$ is equal to 1 , corresponding to a complete inversion of the middle. However, in the usual case, the population inversion is partial and $\mathrm{n}_{\mathrm{sp}}>1$.

The shot noise results in the detection of the received total optical power due to the signal and the power of the ASE noise. It is given by the following equation:

$$
N_{\text {shot }}=2 \cdot e^{2} \cdot B_{e}\left(\frac{G \cdot P_{i n}}{h \cdot v}+n_{s p} \cdot B_{0} \cdot(G-1)\right)
$$

where $B_{e}$ is the electrical bandwidth of the photo-detector.

The noise contribution due to the signal exists as well there is no optical amplifier; this later simply modifies the signal power, then the shot noise power related to this introduces a supplementary shot noise that is associated to the detection of the ASE.

The two intrinsic components related to beat noise are produced at the same time when optical signals and ASE coexist together. The first type of beat noise which is the signal-spontaneous beat noise occurs between optical signals and ASE having frequency close to that of the optical signals. It is given by the following equation:

$N_{s-s p}=4 \cdot \frac{e^{2}}{h \cdot v} \cdot B_{e} \cdot P_{i n} \cdot n_{s p} \cdot G \cdot(G-1)$

The second type which is the spontaneous-spontaneous beat noise occurs between ASEs. It is expressed as follows:

$N_{s p-s p}=e^{2} \cdot\left(2 B_{0}-B_{e}\right) \cdot B_{e} \cdot n_{s p}^{2} \cdot(G-1)^{2}$

The signal-spontaneous beat noise is preponderant for a strong input signal, whereas the spontaneous-spontaneous beat noise is dominating when there is an injection of a small input power. Compared to the shot noise and the signalspontaneous beat noise, the spontaneous-spontaneous beat noise can be significantly minimized by placing an optical filter having a bandwidth $\mathrm{B}_{0}$ after the amplifier.

A convenient way to quantify and characterize the noise and describe its influence on the SOA performance is in term of Noise Figure (NF) parameter. It represents the amount of degradation in the signal to noise ratio caused by amplification process, and it is defined as the ratio between the optical signal to noise ratio (OSNR) of the signal at the input and output of SOA:

$$
N F=\frac{(S / N)_{\text {in }}}{(S / N)_{\text {out }}}
$$

The OSNR of the input signal is given by the following equation [13]:

$$
(S / N)_{i n}=\frac{P_{i n}}{2 \cdot h \cdot v \cdot B_{e}}
$$

The OSNR of the input signal is proportional to the optical power of the input signal, or more specifically to the input number of photons per unit time $\left(\mathrm{P}_{\mathrm{in}} / \mathrm{hv}\right)$. Whereas, the OSNR of the output signal is defined by:

$$
(S / N)_{\text {out }}=\left(\frac{e \cdot P_{i n} \cdot G}{h \cdot v}\right)^{2} \cdot \frac{1}{N_{s h o t}+N_{s-s p}+N_{s p-s p}}
$$

Accordingly, by substituting equations (6), (7), (8), (10) and (11) into (9), the noise figure can be written as follows:

$$
\begin{aligned}
N F= & \frac{1}{G}+2 \cdot n_{s p} \cdot \frac{G-1}{G}+\frac{h \cdot v \cdot B_{0} \cdot n_{s p} \cdot P_{i n} \cdot(G-1)}{P_{o u t}^{2}} \\
& +\frac{h \cdot v \cdot\left(2 B_{0}-B_{e}\right) \cdot n_{s p}^{2} \cdot P_{i n} \cdot(G-1)^{2}}{2 \cdot P_{\text {out }}^{2}}
\end{aligned}
$$

In practical case, the last two terms can be neglected because the ASE power is weak compared to the signal power; else the spontaneous-spontaneous beat noise can be mini- 
mized by placing an optical filter at the output. So the noise figure can be rewritten as [14]:

$N F \approx \frac{1}{G}+2 \cdot n_{s p} \cdot \frac{G-1}{G}$

Since spontaneous emission factor $\left(\mathrm{n}_{\mathrm{sp}}\right)$ is always greater than 1 , the minimum value of NF is obtained for $n_{s p}=1$. So, for large value of gain $(\mathrm{G}>>1)$, the noise figure of an ideal optical amplifier is $3 \mathrm{~dB}$. This is considered as the lowest NF that can be achieved. This implies that every time an optical signal is amplified, the signal to noise ratio is reduced to the half.

\section{SIMULATION RESULTS AND DISCUSSION}

For simulating the noise effect in the SOA device and its impact on the behavior of the structure, we used available OptiSystem software. The physical features of the structure are listed in Table 1. The $\mathrm{CW}$ input signal power ranges from -40 to $10 \mathrm{dBm}$ at wavelength $\lambda=1.55 \mu \mathrm{m}$.

By varying the bias current and the power injected at the SOA input and by modifying its active region length, we measured, using a dual port WDM analyzer, the variation of Gain, NF and OSNR at the output of the structure. Consequently, as shown in obtained results, these parameters will be changed not only with the input signal power variation but also as a function of the SOA length, the confinement factor and the bias current.

Table 1. Device Parameters used in the Simulation

\begin{tabular}{|c|c|c|}
\hline Symbol & Description & Value \\
\hline \hline $\mathrm{I}$ & Bias current & $0.3-0.5 \mathrm{~A}$ \\
\hline$\eta_{\text {in }}$ & Input coupling loss & $3 \mathrm{~dB}$ \\
\hline$\eta_{\text {out }}$ & Output coupling loss & $3 \mathrm{~dB}$ \\
\hline $\mathrm{R}_{1}$ & Input facet reflectivity & $5 \mathrm{e}-005$ \\
\hline $\mathrm{R}_{2}$ & Output facet reflectivity & $5 \mathrm{e}-005$ \\
\hline $\mathrm{L}$ & Active layer length & $1000-1300 \mu \mathrm{m}$ \\
\hline $\mathrm{W}$ & Active layer width & $3 \mu \mathrm{m}$ \\
\hline $\mathrm{d}$ & Active layer height & $0.25 \mu \mathrm{m}$ \\
\hline$\Gamma$ & Optical confinement factor & $0.25-0.45$ \\
\hline $\mathrm{A}$ & Nonradiative recombination coefficient & $3600000001 / \mathrm{s}$ \\
\hline $\mathrm{B}$ & Bimolecular recombination coefficient & $5.6 \mathrm{e}-016 \mathrm{~m} \mathrm{~m}^{\wedge} / \mathrm{s}$ \\
\hline $\mathrm{C}$ & Auger recombination coefficient & $3 \mathrm{e}-041 \mathrm{~m}{ }^{\wedge} 6 / \mathrm{s}$ \\
\hline $\mathrm{V}_{\mathrm{g}}$ & Group velocity & $75000000 \mathrm{~m} / \mathrm{s}$ \\
\hline $\mathrm{m}_{\mathrm{e}}$ & Effective mass of electron in the CB & $4.1 \mathrm{e}-032 \mathrm{Kg}$ \\
\hline $\mathrm{m}_{\mathrm{hh}}$ & Effective mass of a heavy hole in the $\mathrm{VB}$ & $4.19 \mathrm{e}-031 \mathrm{Kg}$ \\
\hline $\mathrm{m}_{\mathrm{hl}}$ & Effective mass of a light hole in the $\mathrm{VB}$ & $5.06 \mathrm{e}-032 \mathrm{Kg}$ \\
\hline $\mathrm{A}_{\mathrm{rad}}$ & Linear radiative recombination coefficient & $100000001 / \mathrm{s}$ \\
\hline $\mathrm{n}_{\mathrm{r}}$ & Active refractive index & 3.7 \\
\hline & & \\
\hline & & \\
\hline
\end{tabular}

The SOA output power versus the input power is shown in Figs. (1) and (2) for cases of bias current $\mathrm{I}=300 \mathrm{~mA}$ and $\mathrm{I}=500 \mathrm{~mA}$, respectively. We can notice that when there is augmentation of SOA active region length, the output power will clearly decreases. In this case, in order to obtain higher output powers, the bias current should be increased. Moreover, there will be a saturation of the output power when the SOA input power is increased.

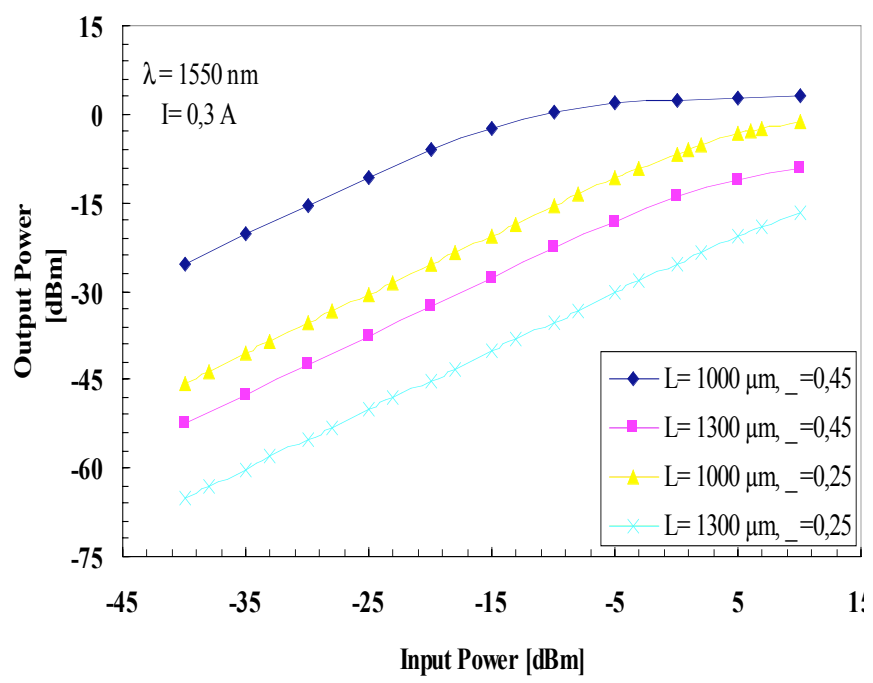

Fig. (1). Output power as a function of input power, SOA length and optical confinement factor: case of bias current $\mathrm{I}=0.3 \mathrm{~A}$.

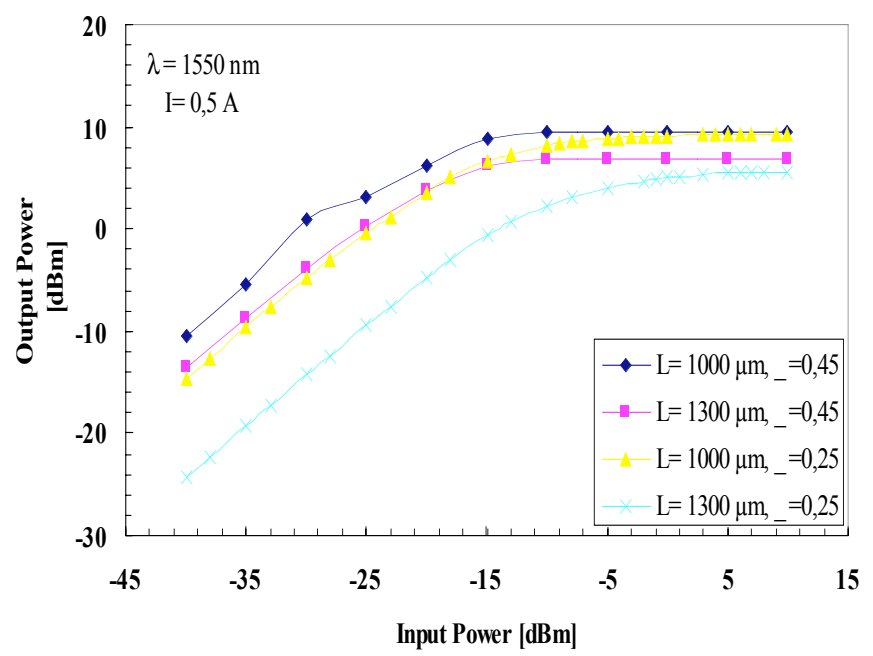

Fig. (2). Output power as a function of input power, SOA length and optical confinement factor: case of bias current $\mathrm{I}=0.5 \mathrm{~A}$.

Results presented in Fig. (3) show that the output noise undergoes changes not only by varying the SOA length but also by modifying the bias current.

The output noise is decreased when we are increasing the input power. It tends to close values for different SOA lengths. At low signal input powers, the output noise will decreases if there is augmentation of SOA length. Besides, we can remark that increasing the bias current has for effect the augmentation of noise especially for long SOAs.

The Fig. (4) displays plots of output OSNR as function of the injected input power and the SOA length. It shows that this parameter is in growth with the input signal power; that is to say if the signal power is weak, ASE power is high and the augmentation of the signal power leads on the diminution 
(a)

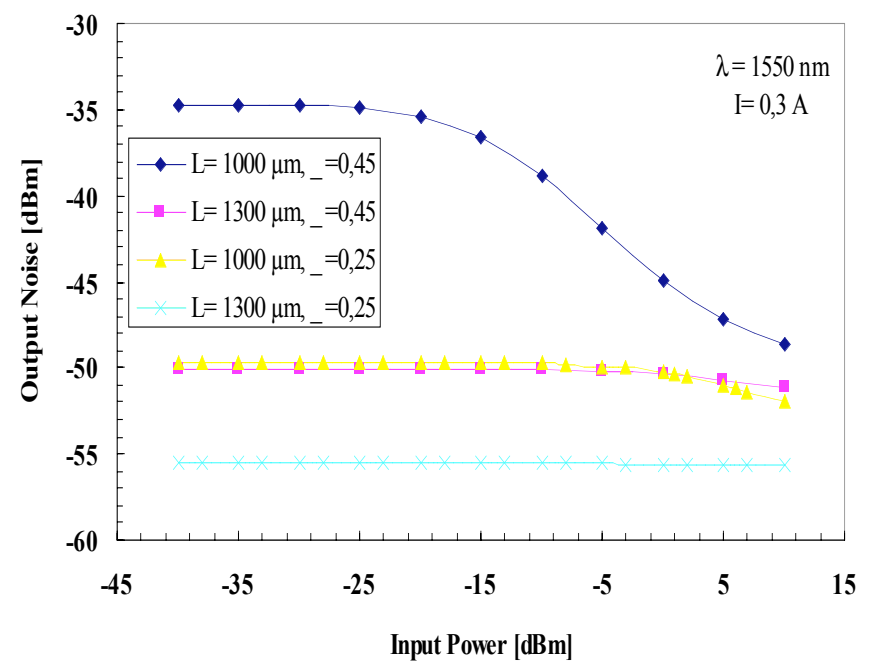

(b)

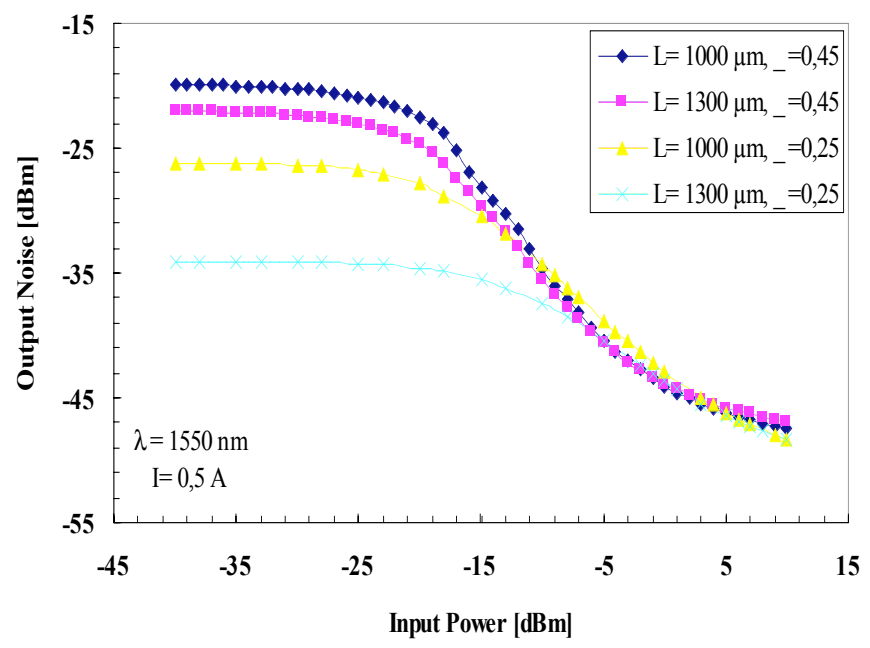

Fig. (3). Representation of the output noise as a function of the input power for various values of the SOA length, the optical confinement factor and the bias current: (a): $\mathrm{I}=0.3 \mathrm{~A},(\mathbf{b}): \mathrm{I}=0.5 \mathrm{~A}$.

of the ASE power. In addition, if the active region length of the structure increases, the output OSNR decreases. This is remarkable for $\mathrm{I}=300 \mathrm{~mA}$, but it is slight when augmenting the bias current to $\mathrm{I}=500 \mathrm{~mA}$. Hence, the bias current should be increased to achieve high values of OSNR at SOA output.

For low input signal powers, Noise Figure parameters undergo small variations; they tend to constant values. From certain threshold point, the augmentation of input power will results an increasing of the NF. This is illustrated in Fig. (5). Besides, long SOAs have an important NF as compared to short ones, which can degrade the performance of the optical network subsystems in which they are integrated. Also, it is shown that the NF is decreased while increasing the bias current.

Plots of NF parameter are distinguished by minima when increasing the input signal power. From these minima, which correspond to certain values of input power, the noise figure undergoes remarkable increasing for different SOA length. These minima, corresponding to bias current $\mathrm{I}=500 \mathrm{~mA}$, are as follows: for long SOA case: $\mathrm{L}=1000 \mu \mathrm{m}, \mathrm{NF}_{\min }=3.15 \mathrm{~dB}$ corresponding to $\mathrm{P}^{\text {in }}{ }_{\min }=-5 \mathrm{dBm}$; wile for $\mathrm{L}=1300 \mu \mathrm{m}$, $\mathrm{NF}_{\min }=5.40 \mathrm{~dB}$, which corresponds to $\mathrm{P}_{\min }^{\mathrm{in}}=-7 \mathrm{dBm}$.

(a)

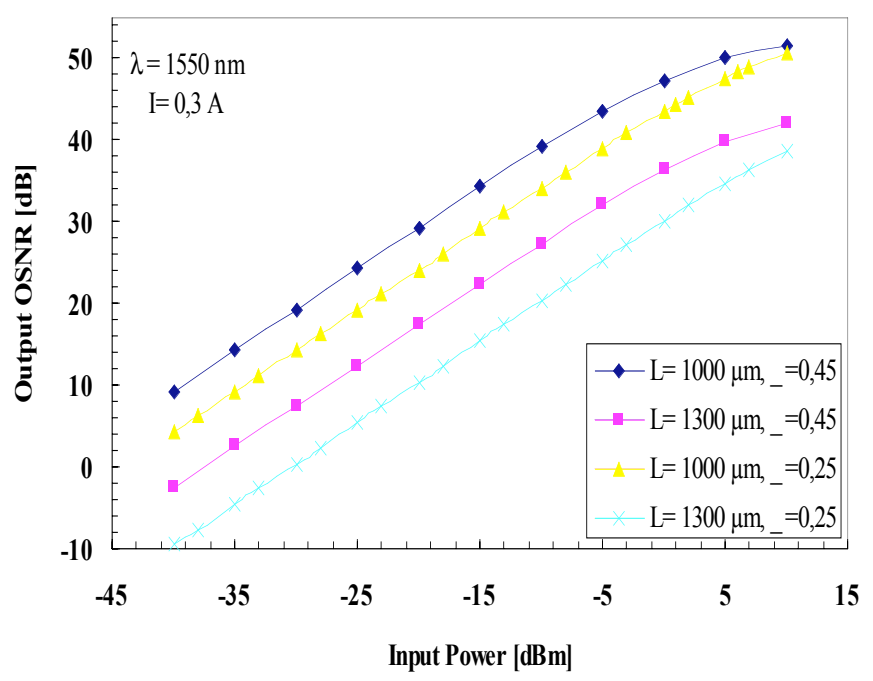

(b)

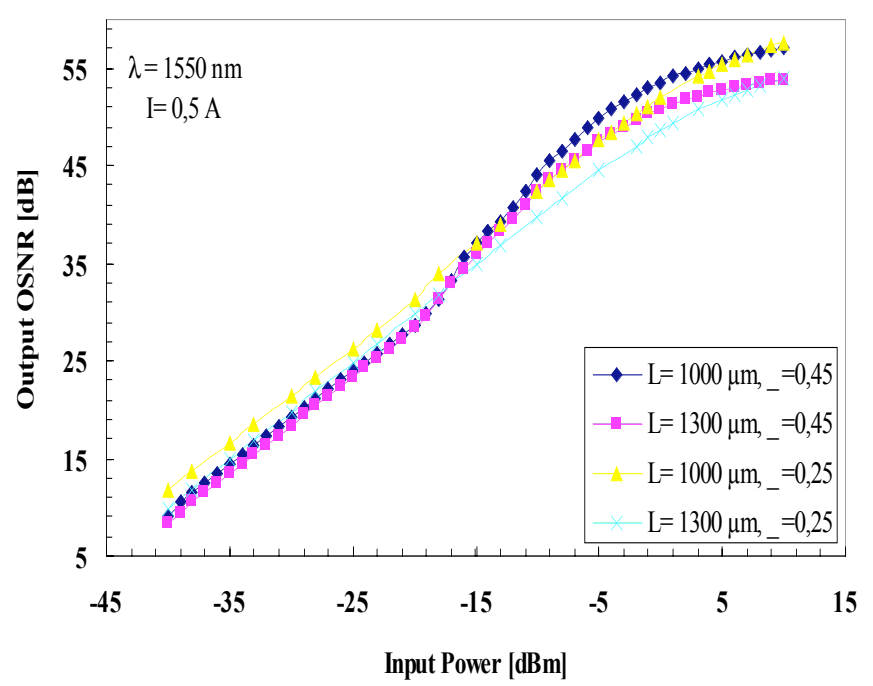

Fig. (4). Curves of output OSNR as a function of input power, SOA length, optical confinement factor and bias current: (a): I= $0.3 \mathrm{~A}$, (b): $\mathrm{I}=0.5 \mathrm{~A}$.

In order to more investigate the noise influence on SOA behavior and to point out its effect on the efficiency of such system configuration, the Gain as a function of the injected signal and the NF versus the gain are plotted for various structure lengths, respectively, in Figs. (6) and (7).

While the input signal power is weak, the carrier density is not modified and the gain keeps constant values. If the input power increases, the stimulated emission becomes preponderant and the carrier density will be minimized; that leads the gain to be saturated. The saturation of the gain will be happened also when the bias current becomes important. Indeed in this case, the carrier density reaches a maximum level in the active region, and then the gain will be stabilized.

Else, we can notice that the gain decreases as the SOA length is increased. This is due to the presence of noise in 
long SOAs. For minimizing its effects, i.e. have high values of gain and low values of noise figure, the bias current shouldn't be chosen to take low value.

(a)

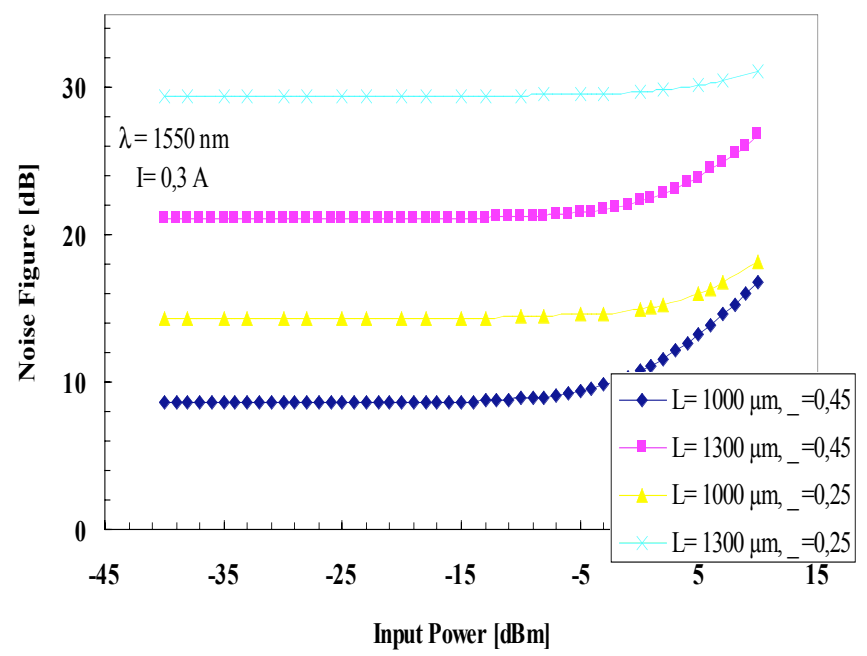

(b)

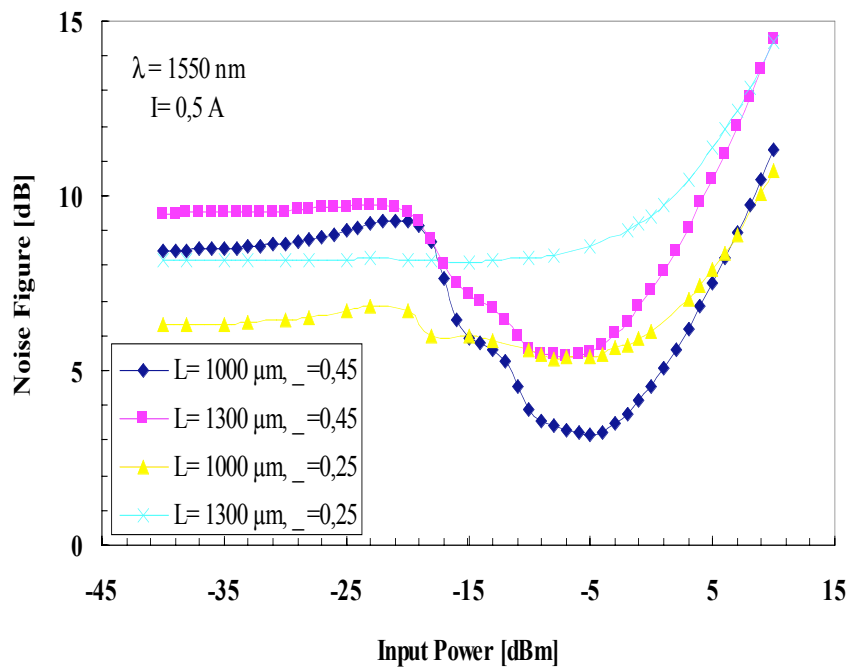

Fig. (5). Representation of the Noise Figure versus the input power for various values of the SOA length, the optical confinement factor and the bias current: $(\mathbf{a})$ : $\mathrm{I}=0.3 \mathrm{~A},(\mathbf{b}): \mathrm{I}=0.5 \mathrm{~A}$.

Else, we can note that the NF decreases with the augmentation of the bias current and, the gain and the ASE power increase in this case. But, the augmentation of the gain is higher than the ASE, for this reason NF decreases.

Moreover, we can see clearly that the gain varies strongly with the optical confinement factor; and while the gain increases, the NF decreases with the augmentation of $\Gamma$.

Although long SOAs have gain dynamics that provide very attractive features of high speed optical signal processing, they present noise able to limit their performance and efficiency. To remedy to this noise effect, using high values of bias current is recommended. It should be pointed out that an accurate choice of the parameters as the input power, the bias current and the SOA length is very important for the determination of the best device operation conditions to achieve the desired functionality based on SOA and to exploit its fast nonlinearities.

(a)

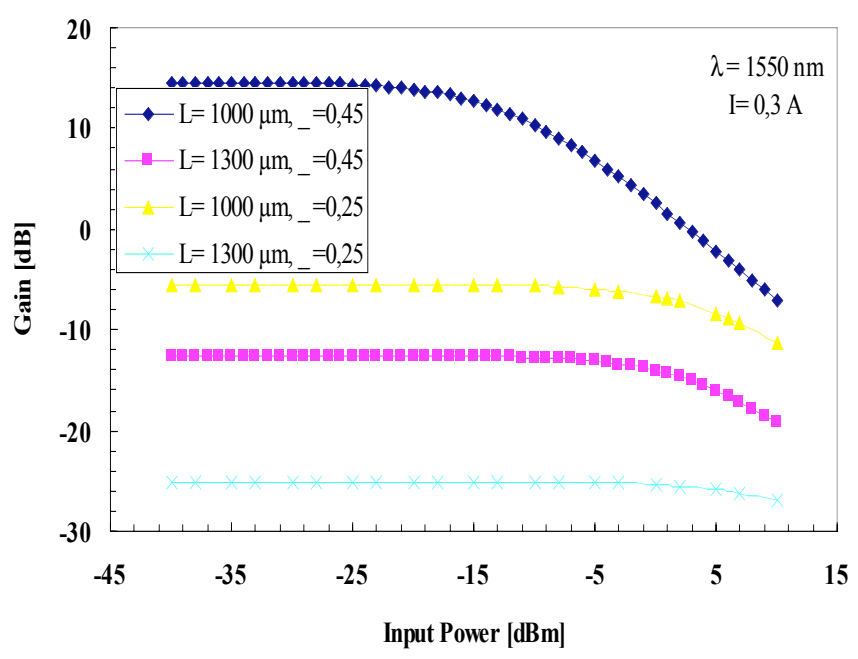

(b)

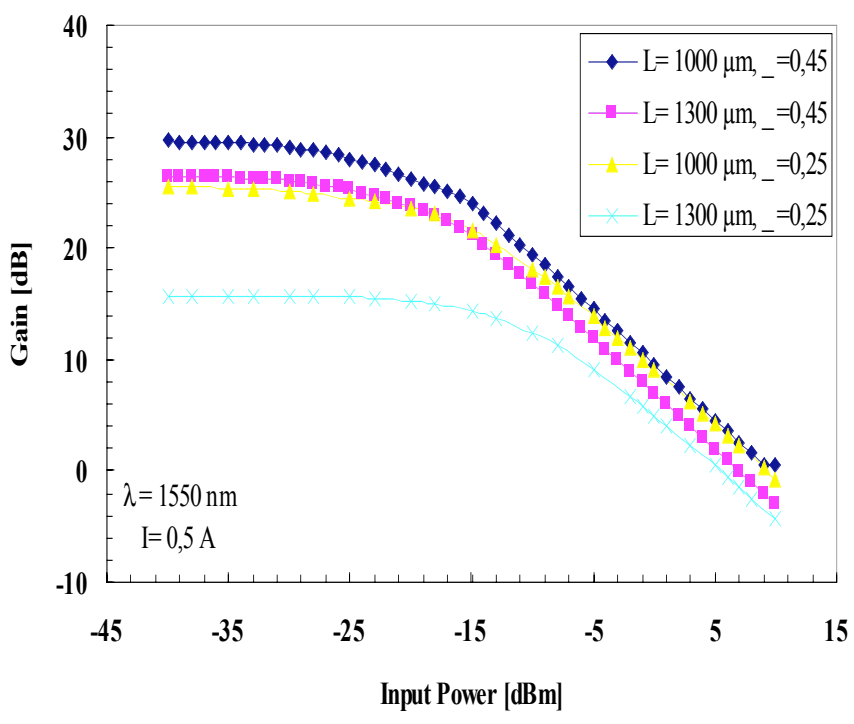

Fig. (6). Evolution of the Gain versus the input power for various values of the SOA length, the optical confinement factor and the bias current: (a): $\mathrm{I}=0.3 \mathrm{~A},(\mathbf{b}): \mathrm{I}=0.5 \mathrm{~A}$.

\section{CONCLUSION}

In this paper, the influence of noise on long SOAs performance was investigated numerically using available simulation software. We analyzed the noise effect and its impact on the device behavior by varying the bias current and the input signal power injected in the structure.

As results, we have shown that the noise is strong in long SOAs and can limit their performance. Moreover, one of the important and decisional parameters to consider, in addition to the input power, is the bias current. By increasing this parameter, the noise figure is minimized. Then, we can remedy to the degradation of the gain and the optical signal to noise ratio at the SOA output. Hence, its fast and high nonlinearities will be also exploited to achieve different functionalities for all-optical signal processing. 
(a)

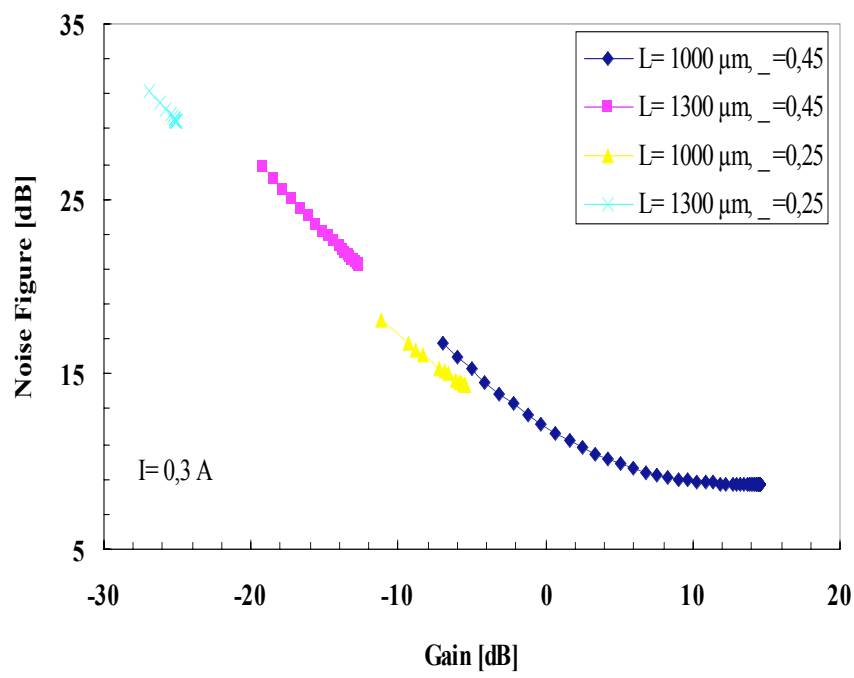

(b)

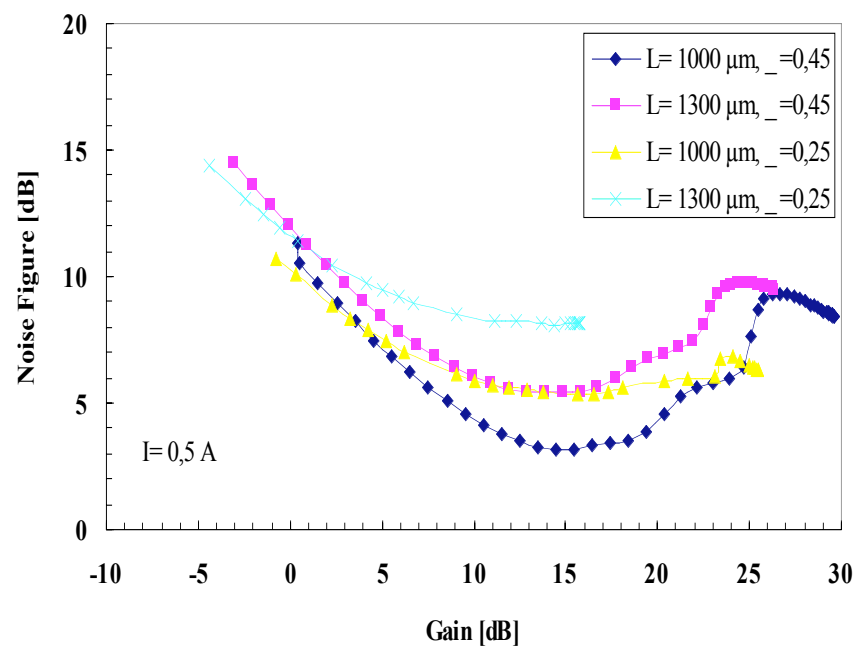

Fig. (7). Representation of Noise Figure versus the Gain for various values of the SOA length, the optical confinement factor and the bias current: (a): I=0.3 A, (b): I=0.5 A.

It should be noticed that the precise influence of SOA noise on system performance depends also on other parameters such as extinction ratio, chirp, modulation format, bitrate and receiver design.

\section{ACKNOWLEDGEMENT}

The authors would like to thank the professor A. Sharaiha and the researchers' team at RESO- ENIB, Brest, France for useful discussions.

\section{REFERENCES}

[1] Politi C, Klonidis D, O'Mahony MJ. Dynamic behavior of wavelength converters based on FWM in SOAs. IEEE J Quantum Electron 2006; 42: 108-25.

[2] Ju H, Zhang S, Waardt H, Tangdiongga E, Khoe GD, Dorren HJS. SOA-based all-optical switch with subpicosecond full recovery. J Opt Express 2005; 13: 942-7.

[3] Masashin U, Munefumi T, Ryo I, Kohsuke N. All optical regeneration using SOA-based polarization discriminated switch injected by transparent assist light. Proc SPIE 2003; 5246: 263-74.

[4] Sharaiha A, Topomondzo J, Morel P. All-optical logic AND-NOR gate with three inputs based on cross-gain modulation in a semiconductor optical amplifier. J Opt Commun 2006; 265: 322-5.

[5] Slovak J, Bornholdt C, Busolt U, Bramann G, Nolting HP, Sartorius B. Dynamic behavior of optically clocked $4 \mathrm{~mm}$ UL-SOA at $40 \mathrm{Gbit} / \mathrm{s}$. Proceedings of SPIE Integrated Optics and Photonic Integrated Circuits, Strasbourg, France 2004; 5451:147-155,.

[6] Bramann G, Wunsche HJ, Busolt U, et al. Two-Wave Competition in Ultralong Semiconductor Optical Amplifiers. IEEE J Quantum Electron 2005; 41: 1260-7.

[7] Shtaif M, Eisenstein G. Calculation of bit error rates in all-optical signal processing applications exploiting nondegenerate four-wave mixing in semiconductor optical amplifiers. IEEE J Lightwave Technol 1996; 14: 2077-96.

[8] Shtaif M, Tromborg B, Eisenstein G. Noise spectra of semiconductor optical amplifiers: relation between semiclassical and quantum descriptions. IEEE J Quantum Electron 1998; 34: 869-78.

[9] Morel P, Sharaiha A, Brenot R, Thédrez B. Wideband gain and noise figure modeling in SOA. Optical Quantum Electron 2006; 38 : 231-6.

[10] Giuliani G, D'Alessandro D. Noise analysis of conventional and gain-clamped semiconductor optical amplifiers. IEEE J Lightwave Technol 2000; 18: 1256-63.

[11] Connelly MJ. Wideband semiconductor optical amplifier steadystate numerical model. IEEE J Quantum Electron 2001; 37: 439-47. Connelly MJ. Semiconductor optical amplifier parameter extraction using a wideband steady-state numerical model and the LevenbergMarquardt method. Proceedings of $4^{\text {th }}$ International Conference of Numerical Simulation of Optoelectronic Devices NUSOD '04; University of California at Santa Barbara; Aug. 24-26. 2004.

[13] Koga M, Matsumoto T. High-gain polarization-insensitive optical amplifier consisting of two serial semiconductor laser amplifiers. J Lightwave Technol 1991; 9: 284-90.

[14] Simon JC, Doussiere P., Pophillat L, Fernier B. Gain and noise characteristics of a $1.5 \mu \mathrm{m}$ near-travelling-wave semiconductor laser amplifier. J Electron Lett 1989; 25: 434-6. 